\title{
ORDER OF OPERATORS DETERMINED BY OPERATOR MEAN
}

\author{
Masaru Nagisa AND Mitsuru Uchiyama
}

(Received July 9, 2013, revised December 20, 2013)

\begin{abstract}
Let $\sigma$ be an operator mean and $f$ a non-constant operator monotone function on $(0, \infty)$ associated with $\sigma$. If operators $A, B$ satisfy $0 \leq A \leq B$, then it holds that $Y \sigma(t A+X) \leq Y \sigma(t B+X)$ for any non-negative real number $t$ and any positive, invertible operators $X, Y$. We show that the condition $Y \sigma(t A+X) \leq Y \sigma(t B+X)$ for a sufficiently small $t>0$ implies $A \leq B$ if and only if $X$ is a positive scalar multiple of $Y$ or the associated operator monotone function $f$ with $\sigma$ has the form $f(t)=(a t+b) /(c t+d)$, where $a, b, c, d$ are real numbers satisfying $a d-b c>0$.
\end{abstract}

1. Introduction. We denote the set of all $n \times n$ matrices over $\mathbb{C}$ by $M_{n}$ and set

$$
H_{n}=\left\{A \in M_{n} ; A^{*}=A\right\} \text { and } H_{n}^{+}=\left\{A \in H_{n} ; A \geq 0\right\},
$$

where $A \geq 0$ means that $A$ is non-negative, that is, the value of inner product

$$
(A x, x) \geq 0 \text { for all } x \in \mathbb{C}^{n} .
$$

Let $J$ be an open interval of the set $\mathbb{R}$ of real numbers. We also denote by $H_{n}(J)$ the set of $A \in H_{n}$ with its spectrum $\operatorname{Sp}(A) \subset J$. A real continuous function $f$ defined on $J$ is said to be operator monotone (in short, $f \in \mathbb{P}(J)$ ) if $A \leq B$ implies $f(A) \leq f(B)$ for any $n \in \mathbb{N}$ and $A, B \in H_{n}(J)$. In this paper, we assume that an operator monotone function is not a constant function.

By the definition of $f \in \mathbb{P}(J)$, for $A, B \in H_{n}, A \leq B$ implies $f(a I+t A) \leq f(a I+t B)$ for a sufficiently small $t>0$, where $a \in J$. Kubo-Ando [5] defined an operator mean $\sigma$ which is a binary operation $X \sigma Y$ for $X, Y \in H_{n}(0, \infty)$, and is given by some $f \in \mathbb{P}(0, \infty)$ with $f(1)=1$ and $f(t)>0$ as follows:

$$
X \sigma Y=X^{1 / 2} f\left(X^{-1 / 2} Y X^{-1 / 2}\right) X^{1 / 2} .
$$

In this paper, we consider the problem whether, for $X, Y \in H_{n}(0, \infty)$ and an operator mean $\sigma$, the following condition for $A, B \in H_{n}$ :

$$
Y \sigma(t A+X) \leq Y \sigma(t B+X) \text { for a sufficiently small } t>0
$$

(correctly, there exists a positive number $\varepsilon$ satisfying that $Y \sigma(t A+X) \leq Y \sigma(t B+X)$ holds whenever $0 \leq t \leq \varepsilon$ ) implies $A \leq B$ or not. Our results are the following:

2010 Mathematics Subject Classification. Primary 47A63; Secondary 15A39.

Key words and phrases. Matrix order, operator mean, operator monotone function, Schur product, Fréchet derivative.

This work was supported in part by Grants-in-Aids for Scientific Research (C)22540220, (C)21540181. 
(1) When $X=c Y$ for some $c>0$, the condition (*) implies $A \leq B$ for any operator mean $\sigma$ ([Corollary 4.2]).

(2) When the associated $f \in \mathbb{P}(0, \infty)$ with $\sigma$ has the form $\frac{a t+b}{c t+d}$, the condition $(*)$ implies $A \leq B$ for any $X, Y \in H_{n}(0, \infty)$ ([Corollary 4.4]).

(3) When the associated $f \in \mathbb{P}(0, \infty)$ with $\sigma$ has not the form $\frac{a t+b}{c t+d}$ and $X$ is not a scalar multiple of $Y$, there exists $A, B, X$ and $Y$ satisfying

$$
A \nless B \text { and } Y \sigma(t A+X) \leq Y \sigma(t B+X)
$$

for a sufficiently small $t>0$ ([Theorem 4.6]).

Combining these facts, we get the following:

TheOREM 1.1. Let $X, Y \in H_{n}(0, \infty)$ and $\sigma$ an operator mean. Then the condition (*) implies $A \leq B$ if and only if $X$ is a positive scalar multiple of $Y$ or the associated operator monotone function $f$ with $\sigma$ has the form

$$
f(t)=\frac{a t+b}{c t+d}, \quad a, b, c, d \in \mathbb{R}, a d-b c>0 .
$$

2. Schur product. For $A \in M_{n}$, we define the linear map $S_{A}$ from $M_{n}$ to $M_{n}$ as follows:

$$
S_{A}(B)=A \circ B \quad\left(B \in M_{n}\right),
$$

where $A \circ B$ means the Schur product of $A$ and $B$, that is,

$$
A \circ B=\left(a_{i j}\right) \circ\left(b_{i j}\right)=\left(a_{i j} b_{i j}\right) .
$$

When $A$ belongs to $H_{n}^{+}$, it is well known that $S_{A}$ is completely positive [2], in particular, $S_{A}\left(H_{n}^{+}\right) \subset H_{n}^{+}$.

We call $A$ of strict rank 1 if there exist $\alpha_{i}, \beta_{i} \in \mathbb{C} \backslash\{0\}(i=1,2, \ldots, n)$ such that

$$
A=\left(\begin{array}{c}
\alpha_{1} \\
\alpha_{2} \\
\vdots \\
\alpha_{n}
\end{array}\right)\left(\begin{array}{llll}
\beta_{1} & \beta_{2} & \cdots & \beta_{n}
\end{array}\right)=\left(\begin{array}{cccc}
\alpha_{1} \beta_{1} & \alpha_{1} \beta_{2} & \cdots & \alpha_{1} \beta_{n} \\
\alpha_{2} \beta_{1} & \alpha_{2} \beta_{2} & \cdots & \alpha_{2} \beta_{n} \\
\vdots & \vdots & \ddots & \vdots \\
\alpha_{n} \beta_{1} & \alpha_{n} \beta_{2} & \cdots & \alpha_{n} \beta_{n}
\end{array}\right)
$$

When $A \in H_{n}^{+}$is of strict rank $1, A$ is represented as follows:

$$
A=\left(\begin{array}{c}
\overline{\gamma_{1}} \\
\overline{\gamma_{2}} \\
\vdots \\
\overline{\gamma_{n}}
\end{array}\right)\left(\begin{array}{llll}
\gamma_{1} & \gamma_{2} & \cdots & \gamma_{n}
\end{array}\right)
$$

where $\gamma_{1}, \gamma_{2}, \ldots, \gamma_{n} \in \mathbb{C} \backslash\{0\}$. The following statement plays an important role in this paper.

Proposition 2.1. For $A=\left(a_{i j}\right) \in H_{n}^{+}$, the following are equivalent:

(1) For $B \in H_{n}, S_{A}(B) \geq 0 \Rightarrow B \geq 0$.

(2) $A$ is of strict rank 1 .

(3) $S_{A}\left(H_{n}^{+}\right)=H_{n}^{+}$. 
(4) $a_{k k}>0$ and $a_{k k} a_{l l}=a_{k l} a_{l k}\left(=\left|a_{k l}\right|^{2}\right)$ for each $k, l$.

Proof. $\quad(1) \Rightarrow(4)$ We assume $a_{k k}=0$. Set $B=\left(b_{i j}\right)$ as follows:

$$
b_{i j}=\left\{\begin{array}{ll}
-1 & \text { if }(i, j)=(k, k) \\
0 & \text { otherwise }
\end{array} .\right.
$$

Since $B \ngtr 0$ and $S_{A}(B)=A \circ B=0 \geq 0$, this contradicts the assumption. So $a_{k k}>0$ for all $k$.

By the positivity of $A$, we have

$$
\left(\begin{array}{cc}
a_{k k} & a_{k l} \\
a_{l k} & a_{l l}
\end{array}\right) \geq 0
$$

in particular, $a_{k k} a_{l l}-a_{k l} a_{l k} \geq 0$. We assume that $a_{k k} a_{l l}-a_{k l} a_{l k}>0$. Then we set $B=\left(b_{i j}\right)$ as follows:

$$
b_{i j}=\left\{\begin{array}{ll}
\frac{\left|a_{k l}\right|}{a_{k k}} & \text { if }(i, j)=(k, k) \\
\frac{\left|a_{k l}\right|}{a_{l l}} & \text { if }(i, j)=(l, l) \\
1 & \text { if }(i, j)=(k, l) \text { or }(l, k) \\
0 & \text { otherwise }
\end{array} .\right.
$$

Since $\left|a_{k l}\right|^{2}=a_{k l} a_{l k}<a_{k k} a_{l l}$, we have $B \ngtr 0$. But we have

$$
(A \circ B)_{i j}= \begin{cases}\left|a_{k l}\right| & \text { if }(i, j)=(k, k) \text { or }(l, l) \\ a_{k l} & \text { if }(i, j)=(k, l) \\ a_{l k} & \text { if }(i, j)=(l, k) \\ 0 & \text { otherwise }\end{cases}
$$

and $A \circ B \geq 0$. This contradicts the assumption. So we have $a_{k k} a_{l l}-a_{k l} a_{l k}=0$ for each $k, l$.

(4) $\Rightarrow$ (2) We set $r_{k}=\sqrt{a_{k k}}>0(k=1,2, \ldots, n)$. By the assumption, for each $k$ and $l$, we can choose $\theta(k, l) \in \mathbb{R}$ such that

$$
a_{k l}=r_{k} r_{l} e^{i \theta(k, l)}
$$

Then we also have $e^{i \theta(k, l)}=e^{-i \theta(l, k)}$ and $e^{i \theta(k, k)}=1$. If we show the relation

$$
e^{i \theta(k, l)} e^{i \theta(l, m)}=e^{i \theta(k, m)}
$$

for any $k, l$ and $m$, then we can see that $A$ is of strict rank 1 as follows:

$$
\left(\begin{array}{c}
r_{1} \\
r_{2} e^{-i \theta(1,2)} \\
\vdots \\
r_{n} e^{-i \theta(1, n)}
\end{array}\right)\left(\begin{array}{llll}
r_{1} & r_{2} e^{i \theta(1,2)} & \cdots & r_{n} e^{i \theta(1, n)}
\end{array}\right)
$$




$$
\begin{aligned}
& =\left(\begin{array}{c}
r_{1} \\
r_{2} e^{i \theta(2,1)} \\
\vdots \\
r_{n} e^{i \theta(n, 1)}
\end{array}\right)\left(\begin{array}{llll}
r_{1} & r_{2} e^{i \theta(1,2)} & \cdots & r_{n} e^{i \theta(1, n)}
\end{array}\right) \\
& =\left(\begin{array}{cccc}
r_{1}^{2} & r_{1} r_{2} e^{i \theta(1,2)} & \cdots & r_{1} r_{n} e^{i \theta(1, n)} \\
r_{2} r_{1} e^{i \theta(2,1)} & r_{2}^{2} e^{i \theta(2,1)} e^{i \theta(1,2)} & \cdots & r_{2} r_{n} e^{i \theta(2,1)} e^{i \theta(1, n)} \\
\vdots & \vdots & \ddots & \vdots \\
r_{n} r_{1} e^{i \theta(n, 1)} & r_{n} r_{2} e^{i \theta(n, 1)} e^{i \theta(1,2)} & \cdots & r_{n}^{2} e^{i \theta(n, 1)} e^{i \theta(1, n)}
\end{array}\right) \\
& =\left(\begin{array}{cccc}
r_{1}^{2} & r_{1} r_{2} e^{i \theta(1,2)} & \cdots & r_{1} r_{n} e^{i \theta(1, n)} \\
r_{2} r_{1} e^{i \theta(2,1)} & r_{2}^{2} & \cdots & r_{2} r_{n} e^{i \theta(2, n)} \\
\vdots & \vdots & \ddots & \vdots \\
r_{n} r_{1} e^{i \theta(n, 1)} & r_{n} r_{2} e^{i \theta(n, 2)} & \cdots & r_{n}^{2}
\end{array}\right)=A .
\end{aligned}
$$

It suffices to show the relation $e^{i \theta(k, l)} e^{i \theta(l, m)}=e^{i \theta(k, m)}$ in the case of each two of $k, l, m$ are different. By the positivity of $A$, we have

$$
\left(\begin{array}{ccc}
a_{k k} & a_{k l} & a_{k m} \\
a_{l k} & a_{l l} & a_{l m} \\
a_{m k} & a_{m l} & a_{m m}
\end{array}\right) \geq 0 .
$$

Since

$$
\begin{aligned}
& \left(\begin{array}{ccc}
a_{k k} & a_{k l} & a_{k m} \\
a_{l k} & a_{l l} & a_{l m} \\
a_{m k} & a_{m l} & a_{m m}
\end{array}\right)=\left(\begin{array}{ccc}
r_{k}^{2} & r_{k} r_{l} e^{i \theta(k, l)} & r_{k} r_{m} e^{i \theta(k, m)} \\
r_{l} r_{k} e^{i \theta(l, k)} & r_{l}^{2} & r_{l} r_{m} e^{i \theta(l, m)} \\
r_{m} r_{k} e^{i \theta(m, k)} & r_{m} r_{l} e^{i \theta(m, l)} & r_{m}^{2}
\end{array}\right) \\
& =\left(\begin{array}{ccc}
r_{k} e^{i \theta(k, l)} & & \\
& r_{l} & \\
& & r_{m} e^{i \theta(m, l)}
\end{array}\right)\left(\begin{array}{ccc}
1 & 1 & \alpha \\
1 & 1 & 1 \\
\bar{\alpha} & 1 & 1
\end{array}\right)\left(\begin{array}{lll}
r_{k} e^{i \theta(l, k)} & & \\
& r_{l} & \\
& & r_{m} e^{i \theta(l, m)}
\end{array}\right)
\end{aligned}
$$

and

$$
\alpha=e^{-i \theta(k, l)} e^{-i \theta(l, m)} e^{i \theta(k, m)},
$$

we have

$$
\left(\begin{array}{lll}
1 & 1 & \alpha \\
1 & 1 & 1 \\
\bar{\alpha} & 1 & 1
\end{array}\right) \geq 0
$$

Then $|\alpha|=1$ and

$$
0 \leq \operatorname{det}\left(\begin{array}{ccc}
1 & 1 & \alpha \\
1 & 1 & 1 \\
\bar{\alpha} & 1 & 1
\end{array}\right)=\alpha+\bar{\alpha}-2
$$

imply $\alpha=1$. So the desired relation is established. 
(2) $\Rightarrow$ (3) When $A$ is of strict rank 1, we construct $A^{\prime} \in H_{n}^{+}$as follows:

$$
A=\left(\begin{array}{c}
\overline{\gamma_{1}} \\
\overline{\gamma_{2}} \\
\vdots \\
\overline{\gamma_{n}}
\end{array}\right)\left(\begin{array}{llll}
\gamma_{1} & \gamma_{2} & \cdots & \gamma_{n}
\end{array}\right), \quad A^{\prime}=\left(\begin{array}{c}
1 / \overline{\gamma_{1}} \\
1 / \overline{\gamma_{2}} \\
\vdots \\
1 / \overline{\gamma_{n}}
\end{array}\right)\left(\begin{array}{llll}
1 / \gamma_{1} & 1 / \gamma_{2} & \cdots & 1 / \gamma_{n}
\end{array}\right) \text {. }
$$

Since $S_{A}\left(H_{n}^{+}\right) \subset H_{n}^{+}, S_{A^{\prime}}\left(H_{n}^{+}\right) \subset H_{n}^{+}$and $S_{A} S_{A^{\prime}}=$ id $=S_{A^{\prime}} S_{A}$, we have that $S_{A}\left(H_{n}^{+}\right)=$ $H_{n}^{+}$.

(3) $\Rightarrow(1)$ When some $(i, j)$-th component of $A$ is equal to 0 , the $(i, j)$-th component of $S_{A}(B)$ is also 0 . So we have all components of $A$ are not equal to 0 . This means that $S_{A}$ is injective. If $S_{A}(B) \geq 0$, then we can choose $C \geq 0$ with $S_{A}(B)=S_{A}(C)$ by the assumption (3). The injectivity of $S_{A}$ implies $B=C \geq 0$.

REMARK 2.2. When $A=A^{*} \in H_{n}, S_{A}\left(H_{n}\right) \subset H_{n}$. Moreover, if $A \in H_{n}$ is of strict rank 1, then $S_{A}\left(H_{n}\right)=H_{n}$ (i.e., $S_{A}$ becomes a bijection of $H_{n}$ ). But there is $A \in H_{n}$ which is not of strict rank 1 and satisfies $S_{A}\left(H_{n}\right)=H_{n}$, for example, $A=\left(\begin{array}{ll}1 & 1 \\ 1 & 2\end{array}\right) \in H_{2}$. In the case of $A \geq 0, S_{A}\left(H_{n}^{+}\right) \subset H_{n}^{+}$and the fact $S_{A}\left(H_{n}^{+}\right)=H_{n}^{+}$is equivalent that $A$ is of strict rank 1 .

3. Fréchet derivatives of operator monotone functions. Let $f$ be an operator monotone function on the open interval $J$ and $A \in H_{n}(J)=\left\{A \in H_{n} ; \operatorname{Sp}(A) \subset J\right\}$. We can choose a positive real number $\varepsilon$ such that $\operatorname{Sp}(A+H) \subset J$ whenever $H \in H_{n}$ and $\|H\|<\varepsilon$. So $f$ is a map from an open neighborhood of $A$ to the real vector space $H_{n}$ and there exists a linear map $T$ from $H_{n}$ to $H_{n}$ satisfying

$$
\lim _{\|H\| \rightarrow 0} \frac{\|f(A+H)-f(A)-T(H)\|}{\|H\|}=0 .
$$

We call this $T$ the (Fréchet) derivative of $f$ at $A$ and denote it by $D f(A)$. For every $B \in H_{n}$, we denote by $D f(A)(B)$ the directional derivative of $f$ at $A$ in the direction $B$, i.e.,

$$
D f(A)(B)=\left.\frac{d}{d t}\right|_{t=0} f(A+t B) .
$$

For a diagonal matrix $\Lambda \in H_{n}(J)$, we can compute as follows (see [1], [2], [4]):

$$
D f(\Lambda)(B)=f^{[1]}(\Lambda) \circ B,
$$

where

$$
\Lambda=\left(\begin{array}{ccc}
\lambda_{1} & & \\
& \ddots & \\
& & \lambda_{n}
\end{array}\right), \quad f^{[1]}(\Lambda)=\left(\begin{array}{ccc}
f^{[1]}\left(\lambda_{1}, \lambda_{1}\right) & \cdots & f^{[1]}\left(\lambda_{1}, \lambda_{n}\right) \\
\vdots & \ddots & \vdots \\
f^{[1]}\left(\lambda_{n}, \lambda_{1}\right) & \cdots & f^{[1]}\left(\lambda_{n}, \lambda_{n}\right)
\end{array}\right)
$$

and

$$
f^{[1]}(\lambda, \mu)=\left\{\begin{array}{ll}
\frac{f(\lambda)-f(\mu)}{\lambda-\mu} & \text { if } \lambda \neq \mu \\
f^{\prime}(\lambda) & \text { if } \lambda=\mu
\end{array} .\right.
$$


The Loewner matrix $f^{[1]}(\Lambda)$ is non-negative since $f$ is operator monotone on $J$. For a general $A \in H_{n}(J)$, we choose a unitary $U \in M_{n}$ and a diagonal matrix $\Lambda \in H_{n}^{+}$satisfying $A=$ $U \Lambda U^{*}$. Then the directional derivatives of $f$ can be represented as follows:

$$
D f(A)(B)=U\left(f^{[1]}(\Lambda) \circ\left(U^{*} B U\right)\right) U^{*} .
$$

If $C \in H_{n}(J), A, B \in H_{n}$ and $C+A, C+B \in H_{n}(J)$, then we have

$$
A \leq B \Rightarrow f(C+A) \leq f(C+B) .
$$

We also have

$$
f(C+t A) \leq f(C+t B) \text { for all } t \in[0,1] .
$$

Since

$$
\frac{f(C+t A)-f(C)}{t} \leq \frac{f(C I+t B)-f(C)}{t} \quad(0<t<1),
$$

it holds $D f(C)(A) \leq D f(C)(B)$ by tending $t$ to 0 . So we have implications as follows:

$$
\begin{aligned}
A \leq B & \Rightarrow f(C+t A) \leq f(C+t B) \quad(0 \leq t \leq 1) \\
& \Rightarrow D f(C)(A) \leq D f(C)(B) .
\end{aligned}
$$

We will consider the problem when the reverse implication holds.

THEOREM 3.1. Let $f$ be an operator monotone function on $J$ and $C \in H_{n}(J)$. Then the following are equivalent:

(1) For $A, B \in H_{n}, D f(C)(A) \leq D f(C)(B)$ implies $A \leq B$.

(2) $f^{[1]}(\Lambda)$ is of strict rank 1 , where $\Lambda$ is a diagonal matrix represented by $U^{*} C U$ for some unitary $U$.

(3) $D f(C)\left(H_{n}^{+}\right)=H_{n}^{+}$.

Proof. First we assume that $C$ is diagonal (i.e., $C=\Lambda$ ). Then we have

$$
D f(C)(A)=D f(\Lambda)(A)=S_{L}(A),
$$

where $L=f^{[1]}(\Lambda)$. In this case, we have (1) $\Leftrightarrow(2) \Leftrightarrow$ (3) by Proposition 2.1.

When $C$ has the form $U \Lambda U^{*}$ ( $\Lambda$ is diagonal and $U$ is unitary), we remark that

$$
D f(C)(A)=U\left(f^{[1]}(\Lambda) \circ\left(U^{*} A U\right)\right) U^{*}=U\left(S_{L}\left(U^{*} A U\right)\right) U^{*} .
$$

Since $U$ is unitary, we have the implication (1) $\Leftrightarrow(2) \Leftrightarrow(3)$.

When $C$ is a scalar operator, we can get the following equivalent conditions and the equivalence of (1) and (2) has been proved in [6]: Theorem 2.2.

THEOREM 3.2. Let $f$ be operator monotone on the open interval $J, A, B \in H_{n}$ and $c \in J$. Then the following are equivalent:

(1) $A \leq B$.

(2) There exists a sequence $\left\{t_{n}\right\}_{n=1}^{\infty}$ satisfying that

$$
t_{n}>0, \lim _{n \rightarrow \infty} t_{n}=0, f\left(c I+t_{n} A\right) \leq f\left(c I+t_{n} B\right) .
$$

(3) $D f(c I)(A) \leq D f(c I)(B)$. 
PROOF. We have already shown (1) $\Rightarrow$ (2) $\Rightarrow$ (3), considering $c I$ as $C$.

(3) $\Rightarrow$ (1) Since $f \in \mathbb{P}(J), f^{\prime}(c)>0$ for $c \in J$. Then we have

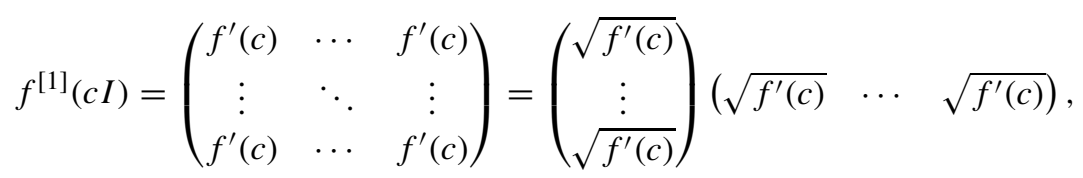

that is, $f^{[1]}(c I)$ is of strict rank 1. By Theorem 3.1, (3) implies (1).

4. Operator means and main results. In this section, we only consider the interval $J$ as $(0, \infty)$. Let $f$ be an operator monotone function on $J$ with $f(1)=1$ and $f(t)>0$ and $\sigma_{f}$ the operator mean associted with $f$. When $X, Y \in H_{n}(J)$ and $A, B \in H_{n}^{+}$with $A \leq B$, we have

$$
Y \sigma_{f}(t A+X) \leq Y \sigma_{f}(t B+X) \text { for all } t \geq 0 .
$$

Our problem is to decide when the condition

$$
Y \sigma_{f}(t A+X) \leq Y \sigma_{f}(t B+X) \quad \text { for a sufficiently small } t>0
$$

implies $A \leq B$.

Proposition 4.1. Let $X, Y \in H_{n}(J)$ and $A, B \in H_{n}^{+}$. If $D f\left(Y^{-1 / 2} X Y^{-1 / 2}\right)\left(H_{n}^{+}\right)=$ $H_{n}^{+}$, then the condition ( $*$ ) implies $A \leq B$.

Proof. By the definition of the mean $\sigma_{f}$, we have the following:

$$
\begin{aligned}
& Y \sigma_{f}(t A+X) \leq Y \sigma_{f}(t B+X) \\
& \Rightarrow f\left(Y^{-1 / 2}(t A+X) Y^{-1 / 2}\right) \leq f\left(Y^{-1 / 2}(t B+X) Y^{-1 / 2}\right) \\
& \Rightarrow \lim _{t \rightarrow 0+} \frac{f\left(Y^{-1 / 2}(t A+X) Y^{-1 / 2}\right)-f\left(Y^{-1 / 2} X Y^{-1 / 2}\right)}{t} \\
& \quad \leq \lim _{t \rightarrow 0+} \frac{f\left(Y^{-1 / 2}(t B+X) Y^{-1 / 2}\right)-f\left(Y^{-1 / 2} X Y^{-1 / 2}\right)}{t} \\
& \Rightarrow D f\left(Y^{-1 / 2} X Y^{-1 / 2}\right)\left(Y^{-1 / 2} A Y^{-1 / 2}\right) \leq D f\left(Y^{-1 / 2} X Y^{-1 / 2}\right)\left(Y^{-1 / 2} B Y^{-1 / 2}\right) .
\end{aligned}
$$

Since $D f\left(Y^{-1 / 2} X Y^{-1 / 2}\right)\left(H_{n}^{+}\right)=H_{n}^{+}$, we have $Y^{-1 / 2} A Y^{-1 / 2} \leq Y^{-1 / 2} B Y^{-1 / 2}$ by Theorem 3.1. This implies $A \leq B$.

In the proof of Theorem 3.2, we have already shown that $D f(c I)\left(H^{+}\right)=H^{+}$for any $c>0$. Noticing the fact $D f(c I)=D f\left(c X^{-1 / 2} X X^{-1 / 2}\right)$, we have the following:

Corollary 4.2. Let $X \in H_{n}(J)$ and $A, B \in H_{n}^{+}$. For any operator mean $\sigma_{f}$ and $c>0$, the condition $(*)$ implies $A \leq B$.

It is well known that the function

$$
f(t)=\frac{a t+b}{c t+d}
$$

is operator monotone on $(-\infty,-d / c)$ and on $(-d / c, \infty)$ if $a d-b c>0$. So $f$ is operator monotone on $J=(0, \infty)$ whenever $a d-b c>0$ and $c d \geq 0$. 
LEMmA 4.3. Let $f(t)=\frac{a t+b}{c t+d}$ with $a d-b c>0$ and $c d \geq 0$. Then we have $D f(X)\left(H_{n}^{+}\right)=H_{n}^{+}$for any $X \in H_{n}(J)$.

PROOF. Since $f$ is operator monotone on $J$, we may show that $f^{[1]}(\Lambda)$ is of strict rank 1 for any diagonal matrix $\Lambda \in H_{n}(J)$. By the argument of Proposition 2.1 (1) $\Rightarrow$ (2), it is sufficient to show that, for any $\lambda, \mu \in J(\lambda \neq \mu)$,

$$
\left(\begin{array}{cc}
f^{\prime}(\lambda) & f^{[1]}(\lambda, \mu) \\
f^{[1]}(\mu, \lambda) & f^{\prime}(\mu)
\end{array}\right)
$$

is of strict rank 1 , that is,

$$
f^{\prime}(\lambda)>0, \quad f^{\prime}(\mu)>0, \quad f^{\prime}(\lambda) f^{\prime}(\mu)=f^{[1]}(\lambda, \mu)^{2} .
$$

By the calculation

and

$$
f^{\prime}(\lambda)=\frac{a d-b c}{(c \lambda+d)^{2}}, \quad f^{\prime}(\mu)=\frac{a d-b c}{(c \mu+d)^{2}}
$$

$$
\begin{aligned}
f^{[1]}(\lambda, \mu) & =\frac{f(\lambda)-f(\mu)}{\lambda-\mu} \\
& =\frac{a d-b c}{(c \lambda+d)(c \mu+d)}=\left(f^{\prime}(\lambda) f^{\prime}(\mu)\right)^{1 / 2},
\end{aligned}
$$

we get $f^{\prime}(\lambda) f^{\prime}(\mu)=f^{[1]}(\lambda, \mu)^{2}$. So we have done.

Combining Proposition 4.1 and Lemma 4.3, we can show the following:

COROLlary 4.4. Let $f(t)=\frac{a t+b}{c t+d}$ with $a d-b c>0$, $c d \geq 0$ and $f(1)=1$, $X, Y \in H_{n}(J)$ and $A, B \in H_{n}^{+}$. Then the condition ( $*$ ) implies $A \leq B$.

In the above statements, we have already used the fact $D f(X)\left(H_{n}^{+}\right)=H_{n}^{+}$as a key tool to get the relation $A \leq B$. But, in many cases, we can not expect that $D f(X)\left(H_{n}^{+}\right)=H_{n}^{+}$ holds.

PROPOSITION 4.5. Let $f$ be operator monotone on $J$ and $X \in H_{n}(J)$ not a scalar matrix. If $D f(X)\left(H_{n}^{+}\right)=H_{n}^{+}$, then the function $f$ is of the form $\frac{a t+b}{c t+d}$.

PROOF. Since $f$ is operator monotone on $J, f$ can be represented as follows:

$$
f(\zeta)=\alpha+\beta \zeta+\int_{-\infty}^{0} g(s, \zeta) d \nu(s), \quad(\operatorname{Im} \zeta>0)
$$

where $\alpha \in \mathbb{R}, \beta \geq 0, g(s, \zeta)=\frac{1+s \zeta}{s-\zeta}$ and $\nu$ is a positive finite measure on $J^{c}=(-\infty, 0]$ ([1], [3], [4]). When $\operatorname{Re} \zeta>0$, the function $g(\cdot, \zeta) \in L^{\infty}\left(J^{c}, v\right)$ and

$$
|g(s, \zeta)| \leq|\zeta|+\left|\frac{\zeta^{2}+1}{s-\zeta}\right| \leq|\zeta|+\frac{|\zeta|^{2}+1}{|\zeta|} .
$$

Using the dominated convergence theorem, for $0<\lambda<\mu$, we can get the following:

$$
f^{\prime}(\lambda)=\beta+\int_{-\infty}^{0} g_{t}(s, \lambda) d v(s)=\beta+\int_{-\infty}^{0} \frac{s^{2}+1}{(s-\lambda)^{2}} d v(s),
$$




$$
f^{[1]}(\lambda, \mu)=\frac{f(\lambda)-f(\mu)}{\lambda-\mu}=\beta+\int_{-\infty}^{0} \frac{s^{2}+1}{(s-\lambda)(s-\mu)} d v(s) .
$$

When $X \in H_{n}(J)$ is not a scalar operator and

$$
\operatorname{Df}(X)\left(H_{n}^{+}\right)=H_{n}^{+},
$$

we show that $f$ has the form $\frac{a t+b}{c t+d}$. We may assume that $\lambda, \mu \in \operatorname{Sp}(X), \lambda \neq \mu$ and

$$
\left(\begin{array}{cc}
f^{\prime}(\lambda) & f^{[1]}(\lambda, \mu) \\
f^{[1]}(\mu, \lambda) & f^{\prime}(\mu)
\end{array}\right)
$$

is of strict rank 1 . The rank of this matrix is 1 implies that $f$ is rational and of degree 1 by Theorem III ([3], page 38).

For convenience of the reader, we prove this statement in [3], here. By above calculation

$$
\begin{gathered}
f^{\prime}(\lambda) f^{\prime}(\mu)-\left(f^{[1]}(\lambda, \mu)\right)^{2}=\left(\beta+\int_{-\infty}^{0} \frac{s^{2}+1}{(s-\lambda)^{2}} d \nu(s)\right)\left(\beta+\int_{-\infty}^{0} \frac{s^{2}+1}{(s-\mu)^{2}} d \nu(s)\right) \\
-\left(\beta+\int_{-\infty}^{0} \frac{s^{2}+1}{(s-\lambda)(s-\mu)} d v(s)\right)^{2} \\
=\beta \int_{-\infty}^{0}\left(\frac{\sqrt{s^{2}+1}}{s-\lambda}-\frac{\sqrt{s^{2}+1}}{s-\mu}\right)^{2} d v(s)+\left(\int_{-\infty}^{0} \frac{s^{2}+1}{(s-\lambda)^{2}} d v(s)\right)\left(\int_{-\infty}^{0} \frac{s^{2}+1}{(s-\mu)^{2}} d v(s)\right) \\
-\left(\int_{-\infty}^{0} \frac{s^{2}+1}{(s-\lambda)(s-\mu)} d v(s)\right)^{2}
\end{gathered}
$$

$=0$.

Applying the Cauchy-Schwarz inequality

$$
\left(\int_{-\infty}^{0} \frac{s^{2}+1}{(s-\lambda)^{2}} d v(s)\right)\left(\int_{-\infty}^{0} \frac{s^{2}+1}{(s-\mu)^{2}} d v(s)\right) \geq\left(\int_{-\infty}^{0} \frac{s^{2}+1}{(s-\lambda)(s-\mu)} d v(s)\right)^{2}
$$

to above identity, we have

$$
\beta \int_{-\infty}^{0}\left(\frac{\sqrt{s^{2}+1}}{s-\lambda}-\frac{\sqrt{s^{2}+1}}{s-\mu}\right)^{2} d v(s)=0
$$

and

$$
\left(\int_{-\infty}^{0} \frac{s^{2}+1}{(s-\lambda)^{2}} d v(s)\right)\left(\int_{-\infty}^{0} \frac{s^{2}+1}{(s-\mu)^{2}} d v(s)\right)=\left(\int_{-\infty}^{0} \frac{s^{2}+1}{(s-\lambda)(s-\mu)} d v(s)\right)^{2} .
$$

Since the equality holds for the inequality, the functions

$$
s \rightarrow \frac{\sqrt{s^{2}+1}}{s-\lambda}, \quad s \rightarrow \frac{\sqrt{s^{2}+1}}{s-\mu}
$$

are linearly dependent in $L^{2}\left(J^{c}, v\right)$ (i.e., the measure $v$ is concentrated on one point $\left.\lambda_{0}\right)$ and $\beta=0$. So we have

$$
f(t)=\alpha+v\left(\left\{\lambda_{0}\right\}\right) \frac{1+\lambda_{0} t}{\lambda_{0}-t},
$$


that is, $f$ has the desired form.

THEOREM 4.6. Let $f$ be operator monotone on $J$ with $f(1)=1$ and not of the form $\frac{a t+b}{c t+d}$. If $X, Y \in H_{n}(J)$ and $X$ is not a scalar multiple of $Y$, then there exist $A, B \geq 0$ such that $A \nless B$ and

$$
Y \sigma_{f}(t A+X) \leq Y \sigma_{f}(t B+X)
$$

for a sufficiently small $t>0$.

PROOF. Since $X$ is not a scalar multiple of $Y$, we may choose a unitary $U$ and a diagonal matrix $\Lambda$ such that

$$
Y^{-1 / 2} X Y^{-1 / 2}=U \Lambda U^{*}
$$

and $\Lambda$ has the form

$$
\left(\begin{array}{lll}
\lambda & & \\
& \mu & \\
& & \ddots
\end{array}\right), \quad \lambda, \mu>0 \text { and } \lambda \neq \mu .
$$

By the assumption for $f$ and Proposition 2.1 and Proposition $4.5, f^{[1]}\left(\left(\lambda_{\mu}\right)\right)$ is not of (strict) rank 1, i.e., $f^{\prime}(\lambda) f^{\prime}(\mu)>f^{[1]}(\lambda, \mu)^{2}$. So we choose $H \in H_{n}$ such that

$$
H=\left(\begin{array}{ccccc}
h_{11} & h_{12} & 0 & \cdots & 0 \\
h_{21} & h_{22} & 0 & \cdots & 0 \\
0 & 0 & 0 & \cdots & 0 \\
\vdots & \vdots & \vdots & \ddots & \vdots \\
0 & 0 & 0 & \cdots & 0
\end{array}\right) \ngtr 0, \quad \operatorname{Df}(\Lambda)(H) \geq 0 .
$$

To restrict the argument to the part of $M_{2}$, we set

$$
H^{\prime}=\left(\begin{array}{ll}
h_{11} & h_{12} \\
h_{21} & h_{22}
\end{array}\right) \ngtr 0, \quad \Lambda^{\prime}=\left(\begin{array}{ll}
\lambda & \\
& \mu
\end{array}\right)>0 .
$$

Since $f^{\prime}(\lambda), f^{\prime}(\mu), f^{[1]}(\lambda, \mu)>0$ and

$$
D f\left(\Lambda^{\prime}\right)\left(H^{\prime}\right)=\left(\begin{array}{cc}
f^{\prime}(\lambda) h_{11} & f^{[1]}(\lambda, \mu) h_{12} \\
f^{[1]}(\mu, \lambda) h_{21} & f^{\prime}(\mu) h_{22}
\end{array}\right) \geq 0,
$$

we have $h_{11}, h_{22}>0$ and may assume that

$$
h_{11} h_{22}<\left|h_{12}\right|^{2}<\frac{f^{\prime}(\lambda) f^{\prime}(\mu)}{f^{[1]}(\lambda, \mu)^{2}} h_{11} h_{22},
$$

in particular, $D f\left(\Lambda^{\prime}\right)\left(H^{\prime}\right)>0$. Put $A^{\prime}, B^{\prime} \geq 0$ as follows:

$$
A^{\prime}=\left(\begin{array}{cc}
0 & 0 \\
0 & \left|h_{12}\right|^{2} / h_{11}-h_{22}
\end{array}\right), \quad B^{\prime}=H^{\prime}+A^{\prime}=\left(\begin{array}{cc}
h_{11} & h_{12} \\
h_{21} & \left|h_{12}\right|^{2} / h_{11}
\end{array}\right) .
$$

Since 


$$
\begin{aligned}
0 & <D f\left(\Lambda^{\prime}\right)\left(H^{\prime}\right)=D f\left(\Lambda^{\prime}\right)\left(B^{\prime}\right)-D f\left(\Lambda^{\prime}\right)\left(A^{\prime}\right) \\
& =\lim _{t \rightarrow 0}\left(\frac{f\left(t B^{\prime}+\Lambda^{\prime}\right)-f\left(\Lambda^{\prime}\right)}{t}-\frac{f\left(t A^{\prime}+\Lambda^{\prime}\right)-f\left(\Lambda^{\prime}\right)}{t}\right) \\
& =\lim _{t \rightarrow 0} \frac{f\left(t B^{\prime}+\Lambda^{\prime}\right)-f\left(t A^{\prime}+\Lambda^{\prime}\right)}{t},
\end{aligned}
$$

we have

$$
f\left(t B^{\prime}+\Lambda^{\prime}\right)-f\left(t A^{\prime}+\Lambda^{\prime}\right) \geq 0
$$

for a sufficiently small $t>0$.

Put

$$
\tilde{A}=\left(\begin{array}{cc}
A^{\prime} & 0 \\
0 & 0
\end{array}\right) \in M_{n}, \quad \tilde{B}=\left(\begin{array}{cc}
B^{\prime} & 0 \\
0 & 0
\end{array}\right) \in M_{n}
$$

and

$$
A=Y^{1 / 2} U \tilde{A} U^{*} Y^{1 / 2}, \quad B=Y^{1 / 2} U \tilde{B} U^{*} Y^{1 / 2} .
$$

Then $A \nless B$ because $A^{\prime} \nless B^{\prime}$. Since

$$
\begin{aligned}
U^{*} Y^{-1 / 2} & \left(Y \sigma_{f}(t B+X)-Y \sigma_{f}(t A+X)\right) Y^{-1 / 2} U \\
= & U^{*} f\left(Y^{-1 / 2}(t B+X) Y^{-1 / 2}\right) U-U^{*} f\left(Y^{-1 / 2}(t A+X) Y^{-1 / 2}\right) U \\
= & U^{*} f\left(t U \tilde{B} U^{*}+Y^{-1 / 2} X Y^{-1 / 2}\right) U-U^{*} f\left(t U \tilde{A} U^{*}+Y^{-1 / 2} X Y^{-1 / 2}\right) U \\
= & U^{*} f\left(U(t \tilde{B}+\Lambda) U^{*}\right) U-U^{*} f\left(U(t \tilde{A}+\Lambda) U^{*}\right) U \\
= & f(t \tilde{B}+\Lambda)-f(t \tilde{A}+\Lambda) \\
= & \left(\begin{array}{cc}
f\left(t B^{\prime}+\Lambda^{\prime}\right)-f\left(t A^{\prime}+\Lambda^{\prime}\right) & 0 \\
0 & 0
\end{array}\right) \geq 0,
\end{aligned}
$$

we have

$$
Y \sigma_{f}(t A+X) \leq Y \sigma_{f}(t B+X)
$$

for a sufficiently small $t>0$.

Acknowledgement. The authors would like to thank the referee for his useful advice. Following his advice, they added the equivalent assertion (4) in Proposition 2.1.

\section{REFERENCES}

[ 1] R. Bhatia, Matrix analysis, Grad. Texts in Math. 169, Springer-Verlag, New York, 1997.

[2] R. Bhatia, Positive definite matrices, Princeton Ser. Appl. Math., Princeton University Press, Princeton, NJ, 2007.

[ 3 ] W. F. Donoghue, JR., Monotone matrix functions and analytic continuation, Springer-Verlag, New YorkHeidelberg, 1974.

[ 4 ] F. HIAI, Matrix analysis: matrix monotone functions, matrix means, and majorization, Interdecip. Inform. Sci. 16 (2010), 139-248.

[ 5 ] F. Kubo And T. Ando, Means of positive linear operators, Math. Ann. 246(1980), 205-224. 
[6] M. Uchiyama, A converse of Loewner-Heinz inequality, geometric mean and spectral order, Proc. Edinb. Math. Soc. (2) 57 (2014), 565-571.

DEPARTMENT OF MATHEMATICS AND INFORMATICS Graduate SCHOOL OF SCIENCE

CHIBA UNIVERSITY

CHIBA, 263-8522

JAPAN

E-mail address: nagisa@math.s.chiba-u.ac.jp
RITSUMEIKAN UNIVERSITY

RESEARCH OFFICE (BKC)

NoJi Higashi 1 CHOME, 1-1 Kusatsu,

SHIGA 525-8577

JAPAN

E-mail address: uchiyama@fc.ritsumei.ac.jp 